\title{
Differential activation of intraepithelial lymphocyte-natural killer cells in chickens infected with very virulent and vaccine strains of infectious bursal disease virus
}

\begin{abstract}
To gain insights into the role of $\mathrm{CD}^{-} / 28.4^{+}$intraepithelial lymphocytes-natural killer (CD3$128.4^{+}$IEL-NK) cells during infectious bursal disease virus (IBDV) infection, characterisation of the cells was performed following infection with different strains of the virus. In vitro treatment with IL-18 or ionomycin/PMA successfully stimulated and activated the cells via a significant increase in the expression of CD69, B-Lec, CHIR-AB1 and NK-lysin. Similarly, chickens infected with the vaccine strain of IBDV also up-regulated the expression of CD69, B-Lec, CHIR-AB1 and NK-lysin in CD3-28.4 IEL-NK cells up to 3 days post infection (dpi) and down-regulated the expression of the inhibitory receptor B-NK at 3 dpi. On the contrary, infection with the very virulent IBDV (vvIBDV) strain lead to a reduced activation of the cells by down-regulating the expression of the CD69, CHIR-AB1 and NKlysin especially at $1 \mathrm{dpi}$. These findings altogether demonstrate the differential activation of CD3 $3 / 28.4^{+}$IEL-NK cells in chicken following infection with the vaccine or very virulent strains of IBDV. The study therefore provides an important clue into the differential pathogenesis of IBDV infection in chicken. Further studies are however required to determine the functional importance of these findings during IBDV vaccination and infection.
\end{abstract}

Keyword: Infectious bursal disease virus; CD3 $/ 28.4^{+}$IEL-NK cells; Activating receptors; Inhibitory receptors 